\title{
Experience from the Thoracic Surgery Residents Association traveling fellowship: Adult and pediatric lung transplantation in Toronto
}

\author{
Taufiek Konrad Rajab, MD, BChir, ${ }^{a}$ Glen Van Arsdell, MD, ${ }^{b}$ and Shaf Keshavjee, MD, MSc ${ }^{c}$
}

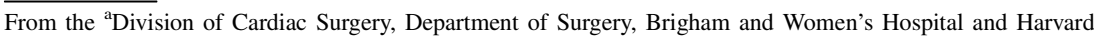
Medical School, Boston, Mass; and the ${ }^{\mathrm{b}}$ Toronto Lung Transplant Program, Department of Surgery, Hospital for Sick Children, and ${ }^{\mathrm{c}}$ Toronto Lung Transplant Program, Department of Surgery, Toronto General Hospital, University of Toronto, Toronto, Ontario, Canada.

The traveling fellowship was funded by the Thoracic Surgery Residents Association.

Disclosures: S.K. is Chief Scientific Officer of Perfusix Canada and XOR Labs Toronto. All other authors have nothing to disclose with regard to commercial support.

This article has been copublished in The Journal of Thoracic and Cardiovascular Surgery and The Annals of Thoracic Surgery.

Received for publication May 15, 2017; revisions received Aug 16, 2017; accepted for publication Aug 16, 2017. Address for reprints: Taufiek Konrad Rajab, MD, BChir, Division of Cardiac Surgery, Department of Surgery, Brigham and Women's Hospital and Harvard Medical School, 75 Francis St, Boston, MA 02115 (E-mail: trajab@bwh.harvard.edu).

J Thorac Cardiovasc Surg 2017;154:2153-4

$0022-5223 / \$ 36.00$

Copyright $@ 2017$ by The American Association for Thoracic Surgery and The Society of Thoracic Surgeons http://dx.doi.org/10.1016/j.jtcvs.2017.08.038
}

Innovations in specific areas of cardiothoracic surgery are spearheaded at a small number of dispersed centers of excellence. The Thoracic Surgery Residents Association (TSRA) and The Society of Thoracic Surgeons have recognized the importance of exposing residents to such innovations by launching the TSRA and Society of Thoracic Surgeons Traveling Fellowship. This fellowship allowed me (T.K.R.) to visit Drs Keshavjee and Van Arsdell in Toronto to learn about their program in adult and pediatric lung transplantation.

The world's first successful adult and pediatric lung transplants were performed in Toronto in 1983 and 1987, respectively. ${ }^{1}$ Ever since, Toronto has been an international leader in this field. Most recently, Dr Keshavjee's group ${ }^{2}$ pioneered ex vivo lung perfusion to evaluate and optimize marginal donor lungs before implantation. Moreover, the

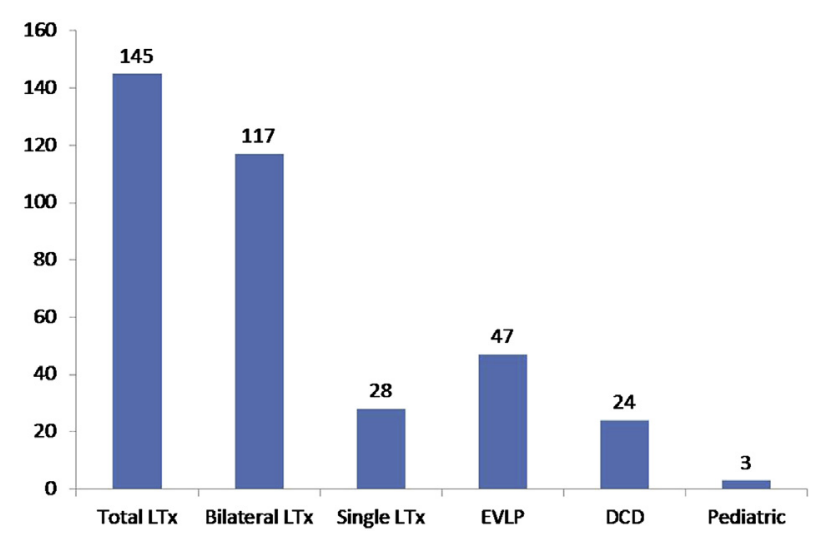

FIGURE 1. Activity of the Toronto Lung Transplant Program in 2016. $L T x$, Lung transplants; $E V L P$, ex vivo lung perfusion; $D C D$, donation after cardiac death. Toronto.

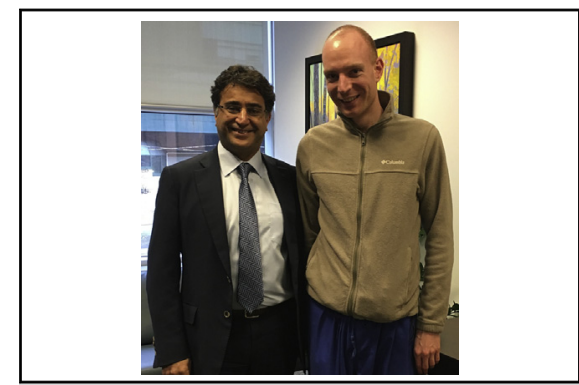

Shaf Keshavjee, MD, MSc (left), and Taufiek Konrad Rajab, MD, BChir (right).

\section{Central Message}

Dr Rajab describes his experience from the TSRA Traveling Fellowship at Toronto General Hospital and the Hospital for Sick Children (SickKids).

program has one of the highest levels of clinical activity in the world (Figure 1). As a result, I had a busy time in

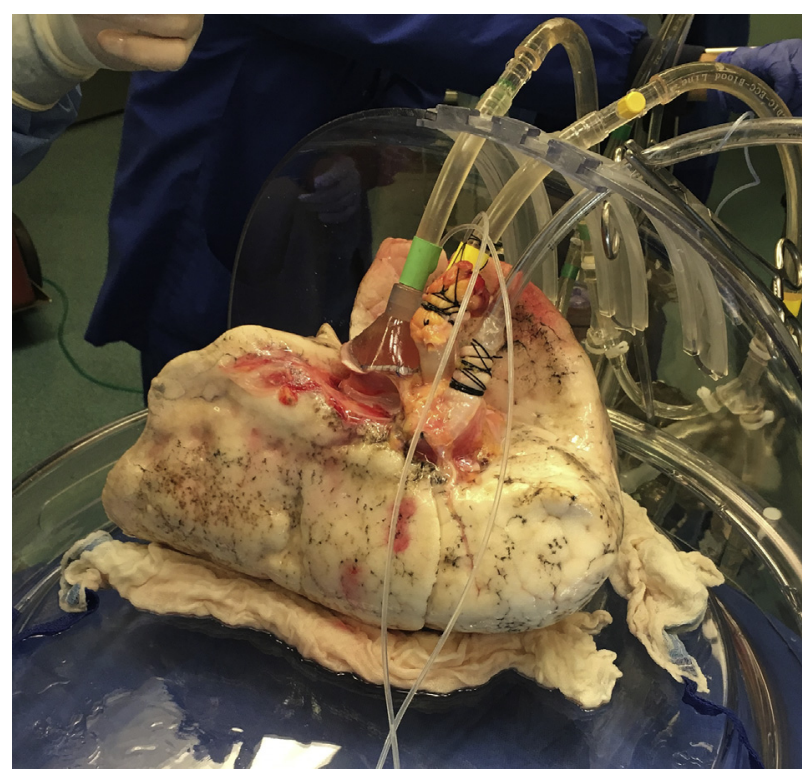

FIGURE 2. Ex vivo lung perfusion to evaluate and optimize a marginal donor lung with 143 minutes of warm ischemic time after donation after cardiac death. S.K. subsequently transplanted this lung into a patient supported by extracorporeal membrane oxygenation for pulmonary allograft rejection. 
I arrived on March 26th and spent the next week attached to the Lung Transplant Program. During this time I was able to attend several procurement runs, observe clinical use of the Toronto ex vivo lung perfusion apparatus for 2 lungs that were marginal as a result of prolonged warm ischemic times after donation after cardiac death (Figure 2), and witness the implantation of one of these lungs. I also attended postoperative care rounds on recipients at Toronto General Hospital and the Hospital for Sick Children (SickKids). Finally, I was able to spend time in the Latner Thoracic Surgery Research Laboratories, where I learned about the program's translational research efforts involving rat and mouse lung transplantation, as well as rat and pig ex vivo lung perfusion experiments.
I am very grateful to the TSRA and The Society of Thoracic Surgeons for their generous traveling fellowship and this unique opportunity to explore my interest in adult and pediatric lung transplantation. In conclusion, I would like to point out that this award should first and foremost be considered recognition of the excellence of the Lung Transplant Program in Toronto.

\section{References}

1. Mendeloff EN. The history of pediatric heart and lung transplantation. Pediatr Transplant. 2002;6:270-9.

2. Cypel M, Yeung JC, Liu M, Anraku M, Chen F, Karolak W, et al. Normothermic ex vivo lung perfusion in clinical lung transplantation. N Engl J Med. 2011;364:1431-40. 